\title{
QMPE: Estimating Lognormal, Wald, and Weibull RT distributions with a parameter-dependent lower bound
}

\author{
ANDREW HEATHCOTE \\ University of Newcastle, Callaghan, Australia \\ SCOTT BROWN \\ University of California, Irvine, California \\ and \\ DENIS COUSINEAU \\ Université de Montréal, Montréal, Québec, Canada
}

\begin{abstract}
We describe and test quantile maximum probability estimator (QMPE), an open-source ANSI Fortran 90 program for response time distribution estimation. ${ }^{1}$ QMPE enables users to estimate parameters for the ex-Gaussian and Gumbel (1958) distributions, along with three "shifted" distributions (i.e., distributions with a parameter-dependent lower bound): the Lognormal, Wald, and Weibull distributions. Estimation can be performed using either the standard continuous maximum likelihood (CML) method or quantile maximum probability (QMP; Heathcote \& Brown, in press). We review the properties of each distribution and the theoretical evidence showing that CML estimates fail for some cases with shifted distributions, whereas QMP estimates do not. In cases in which CML does not fail, a Monte Carlo investigation showed that QMP estimates were usually as good, and in some cases better, than CML estimates. However, the Monte Carlo study also uncovered problems that can occur with both CML and QMP estimates, particularly when samples are small and skew is low, highlighting the difficulties of estimating distributions with parameter-dependent lower bounds.
\end{abstract}

In this paper, we describe and test quantile maximum probability estimator (QMPE), an open-source ANSI standard Fortran 90 program for estimating the parameters $(\Theta)$ of continuous density functions $f(\Theta)$, commonly used to model response time (RT) data. QMPE extends Brown and Heathcote's (2003) quantum maximum likelihood estimation (QMLE) software, which fits only the three-parameter ex-Gaussian distribution, to four new positively skewed distributions: the two-parameter Gumbel distribution (Gumbel, 1958) and the three-parameter shifted Lognormal, shifted Wald, and shifted Weibull distributions. The shifted distributions are bounded below, which makes them attractive as models of RT. Like QMLE, QMPE can fit distributions using continuous maximum likelihood (CML) estimation and quantile maximum probability (QMP2; Heathcote \& Brown, in press; Heath-

This work was supported by Australian Research Council grants to S. Andrews and A.H. and to A.H., B. Hayes, and D. J. K. Mewhort. Thanks to Doug Mewhort for providing computing resources and to several reviewers for their helpful suggestions. Correspondence concerning this article should be addressed to A. Heathcote, School of Behavioural Sciences, Aviation Building, University of Newcastle, University Avenue, Callaghan, 2308 NSW, Australia (e-mail: andrew. heathcote@newcastle.edu.au). cote, Brown, \& Mewhort, 2002) estimation. In the next section, we describe the distribution functions fit by QMPE. We then describe the estimation methods and demonstrate that in cases in which CML estimation fails for shift distributions, QMP estimates are viable. Finally, we report the results of a Monte Carlo study in which CML and QMP estimations in small samples are compared.

\section{QMPE Distribution Functions}

Like Brown and Heathcote's (2003) QMLE program, QMPE fits the ex-Gaussian distribution, a positively skewed distribution produced by the convolution of a normal and exponential distribution (see Heathcote, 1996, for details). The ex-Gaussian has three parameters: the mean $(\mu)$ and standard deviation $(S D ; \sigma>0)$ of the normal component, and the mean of the exponential component $(\tau>0)$. The parameters have a simple relationship to the first three cumulants: the mean $\left(\kappa_{1}\right)$, the variance $\left(\kappa_{2}\right)$, and the third central moment $\left[\kappa_{3}=\right.$ $\left.\int\left(x-\kappa_{1}\right)^{3} f(x) d x\right]$, given by

$$
\begin{aligned}
& \kappa_{1}=\mu+\tau, \\
& \kappa_{2}=\sigma^{2}+\tau^{2},
\end{aligned}
$$

and

$$
\kappa_{3}=2 \tau^{3} .
$$


The third central moment is a measure of skew and can be estimated by the method of moments formula: $\kappa_{3}=\Sigma$ $\left(x_{i}-\bar{x}\right)^{3 / n}$. The Fisher skew measure, $\gamma_{1}=\kappa_{3} / \kappa_{2}^{3 / 2}$, is also often used to quantify distribution asymmetry, since it is a dimensionless quantity that is invariant to scale changes. Figure 1A shows three examples of ex-Gaussian distributions.

Woodworth and Schlosberg (1954) suggested the Lognormal as an empirical approximation to RT distributions. As its name implies, the logarithm of a lognormally distributed random variable is distributed normally (equivalently, an exponentiated normal random variable has a Lognormal distribution). The Lognormal distribution is the asymptotic distribution of the product of random variables (McClelland's [1979] cascade model is an example of a multiplicative model; see Ulrich \& Miller, 1994, for more details). Hence, the Lognormal distribu- tion can be motivated as an approximation to the finishing time of a series of stages with randomly varying rates (West \& Shlesinger, 1990). Breukelen (1995) showed that two well-known parallel models are also compatible with the Lognormal distribution.

The Lognormal distribution is bounded below by zero $(x>0)$ and has parameters corresponding to the normal distribution mean $(\mu)$ and $S D(\sigma>0)$. In order to allow for a lower bound greater than zero, we added a shift parameter, $\theta>0$. The corresponding density, which is defined only for $\theta<x$, is

$$
f(x)=\frac{1}{(x-\theta) \sigma \sqrt{2 \pi}} e^{-\frac{1}{2}\left(\frac{\ln (x-\theta)-\mu}{\sigma}\right)^{2}} .
$$

Ratcliff and Murdock (1976) reported that the shifted Lognormal provided as good a fit as the ex-Gaussian to

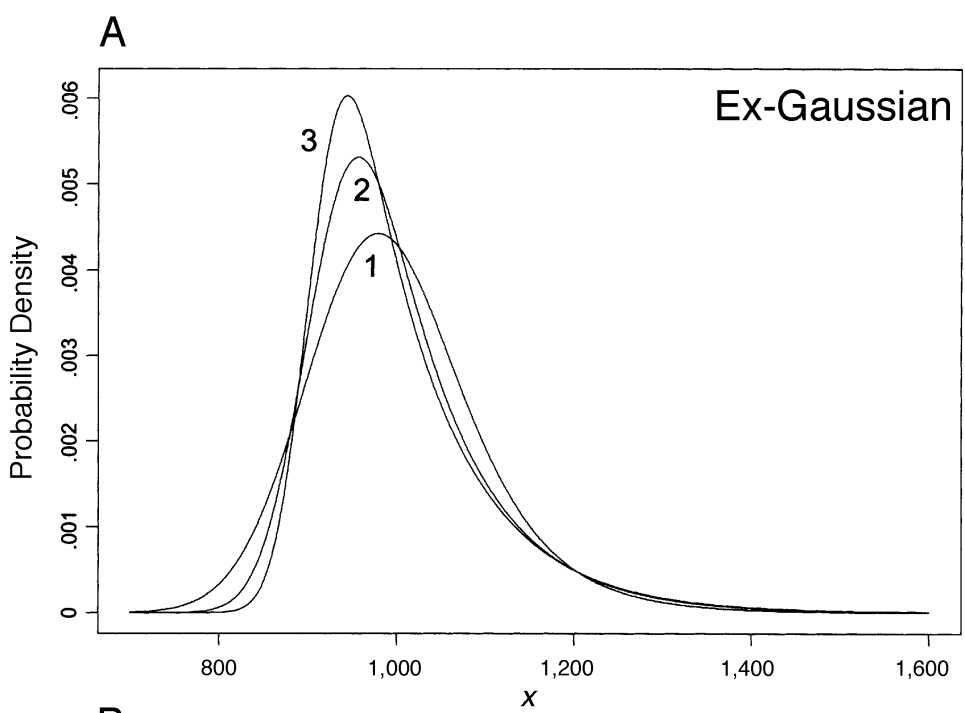

B

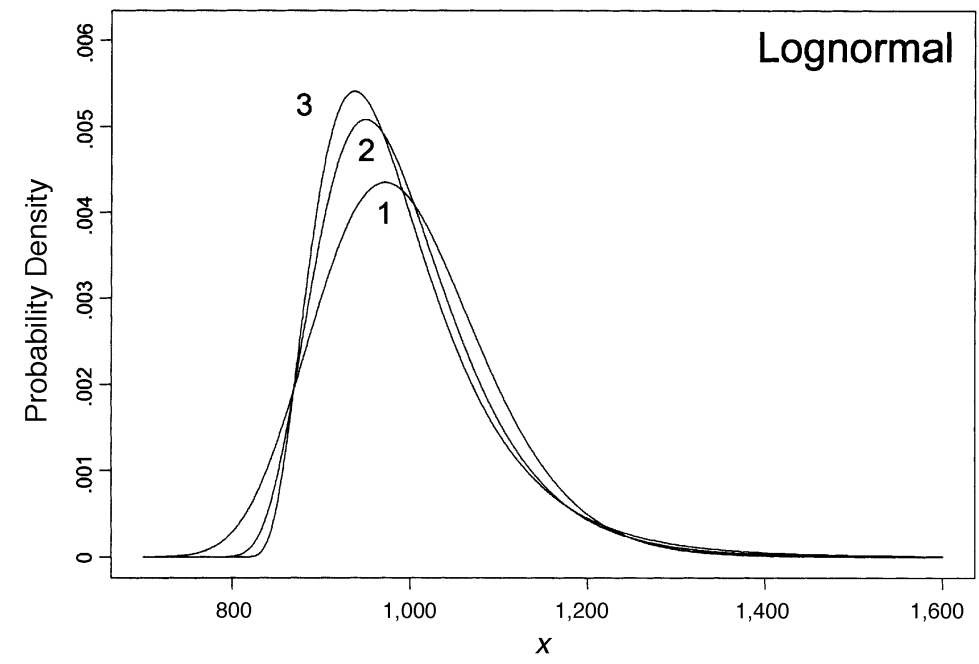

Figure 1. Distributions used in the Monte Carlo study. Table 1 gives the parameters corresponding to the distributions marked 1, 2, and 3 . 

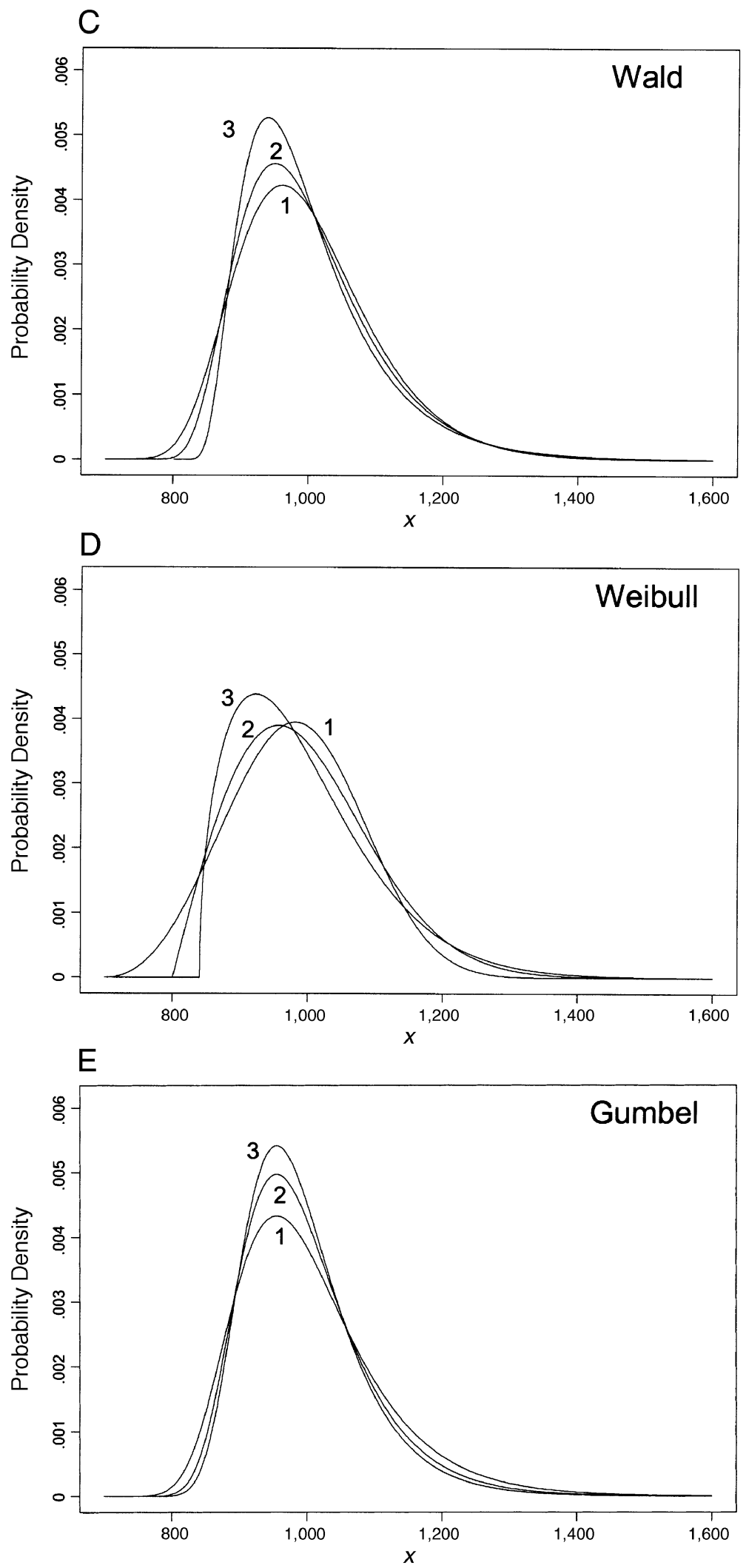

Figure 1 (Continued). 
RT distribution data from recognition memory experiments. Figure 1B shows three examples of the Lognormal density. In order to specify the first three cumulants, it is convenient to define $\omega=\exp \left[\sigma^{2}\right]$ :

and

$$
\begin{aligned}
& \kappa_{1}=\theta+e^{\mu+1 / 2 \sigma^{2}}, \\
& \kappa_{2}=e^{2 \mu} \omega(\omega-1),
\end{aligned}
$$

$$
\kappa_{3}=e^{3 \mu} \omega^{3 / 2}(\omega-1)^{2}(\omega+2) .
$$

The Wald distribution (Wald, 1947) can be motivated as a model of RT by a continuous approximation to the sequential acquisition of information. Suppose that at each time step, identical, normally distributed observations with mean $\mu>0$ are accumulated. A decision to respond is made when the sum exceeds some criterion, $a>0$. Given that $\mu$ is much less than $a$, the number of steps to exceed the criterion is approximately Wald distributed. As described by McGill (1963), in the limit the discrete steps can be replaced by a continuous time variable, resulting in a diffusion process with an exactly Wald-distributed stopping time.

The Wald distribution is bounded below by zero $(x>0)$. We added a shift parameter, $\theta>0$, to allow for a lower bound greater than zero. The corresponding density, which is defined only for $\theta<x$, is

$$
f(x)=\frac{a}{\sqrt{2 \pi(x-\theta)^{3}}} \exp \left\{-\frac{[a-\mu(x-\theta)]^{2}}{2(x-\theta)}\right\} .
$$

Figure 1C shows three examples of the Wald density. The first three cumulants are

and

$$
\begin{aligned}
& \kappa_{1}=\theta+a / \mu, \\
& \kappa_{2}=a / \mu^{3},
\end{aligned}
$$

$$
\kappa_{3}=3 a / \mu^{5} .
$$

The Wald distribution is also used with a different parameterization in terms of its (unshifted) mean, $a / \mu$, and its dispersion, $\lambda=a^{2}$. In this form, the distribution is often called the inverse Gaussian distribution. ${ }^{3}$ When we tested this parameterization with QMPE, we found that parameter estimates, particularly for $\lambda$, were very biased and inefficient. Hence, QMPE uses the diffusion parameterization, which also has the advantage that its parameters are directly interpretable in terms of the information accumulation decision model.

The final two distributions implemented in QMPEthe Weibull and the Gumbel-are related in that they both occur as the asymptotic distribution of the minima of samples from sets of random variables (see Weibull, 1951, and Cousineau, Goodman, \& Shiffrin, 2002, for details). The Weibull arises from the minimum value of samples from random variables that are bounded below by zero, whereas the Gumbel arises from random vari- ables that are unbounded. Both distributions have two parameters, but they differ in that the Gumbel distribution is unbounded, whereas the Weibull distribution is bounded below by zero. As for the other distributions that are bounded below, QMPE adds a shift parameter $(\theta>0)$ to the Weibull.

The Weibull distribution is a power transformation (with exponent $c>0$ ) of an exponential random variable (with mean $\tau>0$ ), as is evident from its density:

$$
f(x)=c \tau^{-c}(x-\theta)^{c-1} e^{-[(x-\theta) / \tau]^{c}} .
$$

As for the other shift distributions, the density is defined only for $\theta<x$. Figure 1D shows three examples of the Weibull density. The cumulants of the Weibull are expressed in terms of the incomplete gamma function $\left[\Gamma(x)=\int_{0}^{\infty} t^{x-1} e^{-t} d t, x>0\right]:$

$$
\begin{aligned}
& \kappa_{1}=\theta+\tau \Gamma\left(c^{-1}+1\right), \\
& \kappa_{2}=\tau^{2}\left[\Gamma\left(2 c^{-1}+1\right)-\Gamma^{2}\left(c^{-1}+1\right)\right],
\end{aligned}
$$

and

$$
\begin{aligned}
\kappa_{3}= & \tau^{3}\left\{\Gamma\left(3 c^{-1}+1\right)-3 \Gamma\left(c^{-1}+1\right) \Gamma\left(2 c^{-1}+1\right)\right. \\
& \left.+2\left[\Gamma\left(c^{-1}+1\right)\right]^{3}\right\} .
\end{aligned}
$$

The Gumbel density is also named the Type I extreme value, the double-exponential, or the Fisher-Tippett distribution. It has only two parameters: one for location $(\mu)$ and one for scale $(\sigma>0)$.

$$
f(x)=\frac{1}{\sigma} \exp \left\{\frac{-(x-\mu)}{\sigma}-\exp \left[\frac{-(x-\mu)}{\sigma}\right]\right\} .
$$

Figure 1E shows three examples of the Gumbel density. Its first three cumulants are $\kappa_{1}=\mu+0.57722 \sigma, \kappa_{2}=(\sigma \pi)^{2} / 6$, and $\kappa_{3}=2.40412 \sigma^{3}$. The Fisher skew of the Gumbel is fixed $\left(\gamma_{1}=1.13955\right)$, so it does not have the flexibility to model changes in RT distribution skew. Although the Gumbel cannot be a general model of RT distribution, it was included in QMPE because it might have utility in special cases. Although we briefly report estimation results for the Gumbel, our main focus is on estimation of shift distributions.

\section{Likelihood and Shifted Distributions}

The likelihood of a sample is the joint probability of the sample according to the distribution model. When the observations making up the data $\left(x_{\mathrm{i}}, i=1,2, \ldots 3, n\right)$ are sampled independently, the joint probability is given by the product of the probabilities for each observation. With maximum likelihood methods, model parameter estimates that maximize likelihood are chosen. However, when applied to continuous distributions, the conventional approach to maximum likelihood estimation maximizes the product of the densities for each observation, 
$f\left(x_{i}, \Theta\right)$, rather than the product of their probabilities. This procedure is justified using an approximation to the probability $(\mathrm{R})$ of each observation:

$$
\begin{array}{rl}
p\left(x_{i}-h_{i} / 2 \leq X \leq x_{i}+h_{i} / 2\right) & = \\
\int_{x_{i}-h_{i} / 2}^{x_{i}+h_{i} / 2} & f(x, \Theta) d x \approx f(x, \Theta) h_{i} .
\end{array}
$$

Two assumptions must hold to ensure that maximizing the product of the densities,

$$
\prod_{i=1}^{n} f\left(x_{i}, \theta\right),
$$

is equivalent to maximizing the joint probability. First, the $h_{\mathrm{i}}(>0)$ must be small for the approximation to be accurate, and the approximation can be made to be exact as the $h_{\mathrm{i}}$ tend to zero. Second, the $h_{\mathrm{i}}$ must be independent of $\Theta$, so that the $h_{\mathrm{i}}$ can be ignored in the maximization. We will refer to this approximation as CML. For computational reasons, estimates are usually found by maximizing the log-likelihood (i.e., the sum of the logarithms of the densities),

$$
\sum_{i=1}^{n} \ln f\left(x_{i}, \Theta\right),
$$

which is equivalent to maximizing the product of the logarithms of the densities.

Unfortunately, the assumptions underlying the CML approximation can fail in some cases, such as when the distribution's range depends on its parameters, as is the case for shift distributions. When the shift parameter $(\theta)$ equals the smallest observation, CML log likelihood is infinite, since the density for the smallest observation $x_{1}$ is zero (without loss of generality, we assume the observations are ordered; e.g., $x_{1} \leq x_{2} \leq \cdots \leq x_{n}$ ). In this case, CML estimates of the other parameters become inconsistent, in the sense that they do not tend to their true values as sample size increases. The singularity associated with $\theta=x_{1}$ is not a problem for iterative estimation methods if (1) log likelihood has a local maximum (say, at $\theta_{\mathrm{L}}<x_{1}$ ), which yields consistent parameter estimates, and (2) if the singularity is disconnected (i.e., log likelihood decreases as $\theta$ approaches $x_{1}$ from below on the interval $\left.\theta_{\mathrm{L}} \leq x<x_{1}\right)$. The singularity has been shown to be disconnected for the shifted Wald distribution (Cheng \& Amin, 1981).

For the shifted Lognormal and Weibull distributions, the singularity is not always disconnected, so inconsistent estimates can be obtained when the log likelihood is maximized by iterative methods. This problem, which we will call the unbounded likelihood problem, occurs particularly for parameter values for which the distribution is highly skewed and has a sharply increasing leading edge. For the Lognormal distribution, the unbounded likelihood problem is rarely a practical concern, given that the increasing region is usually very small (e.g., Giesbrecht \& Kempthorne, 1976, Table 1), and a local maximum that produces consistent parameter estimates usually exists outside this region. Given good starting point estimates, iterative methods will converge on this local maximum and provide consistent parameter estimates, which are sometimes called local maximum likelihood estimates.

For the Weibull distribution, however, the unbounded likelihood problem can cause more severe difficulties in

\begin{tabular}{|c|c|c|c|c|c|c|c|}
\hline Distribution & Set & $\mu$ & $\sigma$ & $\tau$ & $M$ & $S D$ & $\gamma_{1}$ \\
\hline \multirow[t]{4}{*}{ Ex-Gaussian } & 1 & 929.289 & 70.711 & 70.711 & 1,000 & 100.000 & 0.7071 \\
\hline & 2 & 910.557 & 44.721 & 89.443 & 1,000 & 100.000 & 1.4311 \\
\hline & 3 & 905.132 & 31.623 & 94.868 & 1,000 & 100.000 & 1.7076 \\
\hline & & $\theta$ & $\sigma$ & $\mu$ & $M$ & $S D$ & $\gamma_{1}$ \\
\hline \multirow[t]{4}{*}{ Lognormal } & 1 & 470 & 0.18 & 6.25 & 996.47 & 95.538 & 0.5504 \\
\hline & 2 & 745 & 0.36 & 5.45 & 993.34 & 92.379 & 1.1674 \\
\hline & 3 & 800 & 0.48 & 5.15 & 993.49 & 98.488 & 1.6590 \\
\hline & & $\theta$ & $\mu$ & $a$ & $M$ & $S D$ & $\gamma_{1}$ \\
\hline \multirow[t]{4}{*}{ Wald* } & 1 & 625 & 0.1886 & 70.711 & 1,000 & 102.698 & 0.8216 \\
\hline & 2 & 725 & 0.1626 & 44.721 & 1,000 & 101.973 & 1.1124 \\
\hline & 3 & 800 & 0.1414 & 28.284 & 1,000 & 100.000 & 1.5000 \\
\hline & & $\theta$ & $\tau$ & $c$ & $M$ & $S D$ & $\gamma_{1}$ \\
\hline \multirow[t]{4}{*}{ Weibull } & 1 & 700 & 315 & 3.2 & 982.13 & 96.772 & 0.1064 \\
\hline & 2 & 800 & 220 & 2.0 & 994.97 & 101.915 & 0.6311 \\
\hline & 3 & 840 & 170 & 1.5 & 993.47 & 104.200 & 1.0720 \\
\hline & & $\mu$ & $\sigma$ & - & $M$ & $S D$ & $\gamma_{1}$ \\
\hline \multirow[t]{3}{*}{ Gumbel } & 1 & 955 & 85 & - & $1,004.06$ & 109.017 & 1.1396 \\
\hline & 2 & 955 & 74 & - & 997.71 & 94.909 & 1.1396 \\
\hline & 3 & 955 & 68 & - & 994.25 & 87.213 & 1.1396 \\
\hline
\end{tabular}

Table 1

Parameters of Distributions Used in the Monte Carlo Study and Associated Moment Statistics

*The samples were generated using the inverse Gaussian parameterization, with $(M, \lambda)=$ $(375,5000),(275,2000)$, and $(200,800)$ for Sets $1-3$, respectively. 
the estimation of the highly skewed distributions produced by small values of the Weibull shape parameter, $c$. For $c=1$, the Weibull distribution is equivalent to the exponential distribution, which has a sharp and discontinuous leading edge. For $c<1$, even more skewed distributions with a shape similar to the exponential are obtained. For $c>1$, the distribution becomes less skewed and the increase of the leading edge is more gradual. Symmetry occurs for $c \approx 3.6$, and skew then becomes negative as $c$ increases, approaching a lower bound Fisher skew $\approx 1.14$. For $c>2$, the singularity is disconnected. For $c<2$, the singularity is connected, but when $c>1$ a local maximum usually exists which produces consistent parameter estimates (Cheng \& Amin, 1983). When $c<$ 1 , no local minimum exists, so iterative estimation results in a shift estimate $\theta=x_{1}$ and inconsistent estimates of the other parameters. Similar conditions apply to the shifted gamma, another distribution commonly used to model RT which also has the exponential distribution as a special case, for exactly the same values of its shape parameter (Cheng \& Amin, 1983). Although not described here, a newer version of QMPE also fits the shifted gamma distribution, and its parameter estimates behave similarly to those of the shifted Weibull.

Cheng and Amin (1983) and, independently, Ranneby (1984) suggested a solution to the unbounded likelihood problem, called the maximum product of spacings (MPS) method. MPS, like CML, obtains parameter estimates by maximizing a goodness-of-fit (objective) function. The MPS objective function is proportional to a special case of the QMP objective function, and so produces identical estimates. As defined by Heathcote et al. (2002), QMP estimates are obtained by maximizing the multinomial log likelihood:

$$
\sum_{j=1}^{m} N_{j} \ln \left(D_{j}\right), \text { where } D_{j}=\int_{\hat{q}_{j-1}}^{\hat{q}_{j}} f\left(x_{i}, \Theta\right) d t .
$$

The $\hat{q}_{j}, j=1, \ldots m-1$ are quantile estimates, $\left(\hat{q}_{o}, \hat{q}_{m}\right)$ equals the domain of the distribution (which might depend on $\Theta$ ), and each interquantile range $\left(\hat{q}_{j-1}, \hat{q}_{j}\right)$ contains $N_{j}$ observations (in general, $N_{j}$ may not be an integer).

The MPS estimator is a special case of $\mathrm{QMP}^{4}$ in which order statistics (i.e., $x_{\mathrm{i}}$ ) are used to estimate quantiles, $N_{j}=1$ and $m=n$. Titterington (1985) suggested a modified version of the MPS objective function that is proportional to the QMP1 objective function examined by Heathcote et al. (2002). By QMP1, we mean estimates obtained by maximizing Equation 1 and based on $\hat{q}_{j}=$ $\left(x_{j}+x_{j+1}\right) / 2, j=1, \ldots n-1$, and $N_{j}=1$. Heathcote et al. showed that QMP1 produced more efficient and less biased estimates than CML for the ex-Gaussian distribution. They also examined QMP4 estimates, in which the data set is reduced to a set of $(n / 4)-1$ equally spaced quantile estimates [for $n=4 m, \hat{q}_{j}=\left(x_{4 j}+x_{4 j+1}\right) / 2$, and $\left.N_{j}=4\right]$, and found estimation performance similar to that of CML, despite the fact that QMP4 is clearly not a sufficient estimator (i.e., it does not use all of the information contained in the data set).

Although Titterington (1985) suggested that MPS can be viewed as maximum likelihood estimation for grouped data, it is important to acknowledge that the equivalence is only approximate in finite samples, because Equation 1 does not take into account the error associated with quantile estimates. However, when the range of the distribution is not parameter dependent, MPS and CML are asymptotically equal, as was shown by Cheng and Amin (1983), so in this case MPS has all of the asymptotic sufficiency, consistency, and efficiency properties of CML. When the range of the distribution is parameter dependent, CML and MPS can behave quite differently. Importantly, both the original version of MPS and Titterington's variation (i.e., QMP1) differ from CML in that they are not subject to the unbounded likelihood problem (Cheng \& Iles, 1987). Hence, they continue to give consistent and efficient estimates even when CML completely fails. In fact, these estimates can become "superefficient," in the sense that estimation error decreases as sample size $(n)$ increases at a rate faster than $n^{-1 / 2}$.

In summary, it is clear that the unbounded likelihood problem can cause CML to fail completely in cases in which QMP continues to work well. It might be argued that such cases are of little interest for RT distribution fitting, as RT distributions rarely have a sufficiently sharp leading edge or degree of skew. We are not aware of any systematic investigation on this point, and we caution that sampling error may cause the problem to occur in small samples even if the true distribution comes from a parameter region where CML does not fail. In any case, there is little point in comparing the estimation performance of CML and QMP in such cases, given that QMP will necessarily be superior. The parameters for the Monte Carlo study were chosen to avoid distributions associated with CML failure.

\section{MONTE CARLO STUDY}

The Monte Carlo study was modeled after the study reported by Heathcote et al. (2002). It had three aims: (1) to test the QMPE code extensively, (2) to compare the estimation performance of CML and QMP, and (3) to compare estimation performance among the five distributions fit by QMPE. Relatively small sample sizes were used ( $n \mathrm{~s}=40,80$, and 160$)$ in order to investigate performance under demanding and realistic conditions. QMP estimation was performed using both QMP1 and QMP4.

For each distribution, three sets of parameter values (presented in Table 1) were used. (Figure 1 illustrates the corresponding densities.) The parameters for the exGaussian distribution were the three sets with medium levels of skew used by Heathcote et al. (2002). The choice of parameters for the other distributions was guided by fitting them to large samples from the three ex-Gaussian distributions, so that the results are approximately compara- 
ble across distributions. As Table 1 shows, this procedure resulted in a fairly good match on means and $S D$ s. Fisher skew varied more between distribution types but covered approximately the same range, except for the Weibull distribution, where skew was generally lower, and the Gumbel distribution, where skew is fixed. The smallest value of the Weibull shape parameter investigated $(c=1.5)$ was large enough to avoid CML failure due to the unbounded likelihood problem even in the smallest samples.

Examination of Figure 1 indicates that shift estimation in the least skewed cases of the Lognormal and Wald distributions (labeled " 1 " in the figure) will be challenging, because they have long, thin left tails. Such tails make estimation of the shift parameters difficult because samples near the lower bound are rare, and the sampled values of the first order statistic $\left(x_{1}\right)$ are highly variable. As a result, shift estimates are likely to be biased upward and to be more variable for these cases in the Monte Carlo study, particularly in small samples.

\section{Method}

For each distribution type and for the nine combinations of sample size and parameter set, 10,000 replicates were fit with the same samples fit by CML, QMP1, and QMP4. The simulated samples were obtained using random-number generators provided by the S-plus statistical package, ${ }^{5}$ and were rounded to the nearest integer.

QMPE uses the same numerical methods as QMLE (see Brown \& Heathcote, 2003, for more details). CML and QMP estimates are obtained by a conjugate gradient optimization algorithm. This algorithm requires analytic expressions for the gradient of the objective function. However, QMPE requires only analytic gradients for the density; gradients for CML and QMP are automatically computed from the density gradients. Once search is complete, analytic expressions for the Hessian (second derivative matrix) of the density are used to estimate approximate parameter standard errors and correlations (see Brown \& Heathcote, 2003, for a proof that these estimates are asymptotically correct for QMP). Although derivative free optimization methods are available, we have found that analytic gradients greatly speed estimation and that analytic Hessians result in better standard error and correlation estimates.

QMPE automatically obtains starting points for optimization by substituting method of moments estimates of cumulants into the equations relating cumulants and parameters. However, this approach fails when sample estimates of skew are negative. In such cases, heuristics are used to estimate starting points. For the three distributions with shift parameters, the heuristic estimates the shift as slightly smaller than the minimum value in the sample (e.g., $\hat{\theta}=p \times x_{1}$, where $0<p<1$ is an appropriately chosen constant), then solves for the other parameters using the first two moments calculated on $\mathbf{x}-\hat{\theta}$. The heuristic is always used for the Lognormal, which we found rarely works with the full method of moments approach. Since it has only two parameters, Gumbel start- ing points are obtained from only the first two cumulants. Users can also supply their own starting points.

Good automatic starting point estimates are essential when large numbers of conditions must be fit, and particularly for the shifted distributions when only the local CML solution is useful. QMPE's starting point heuristics were fine tuned throughout the course of the Monte Carlo study. We have also found them to work well in real RT data.

The stopping criteria for optimization were set at a proportional objective function exit tolerance of $10^{-9}$ and a proportional parameter change tolerance of $10^{-5}$, and the maximum number of search iterations was fixed at 250 (see Brown \& Heathcote, 2003, for details on these settings), resulting in parameter estimates accurate to more than four significant figures. For all parameters bounded below by zero, QMPE sets the objective function to a low value when the estimate is less than $10^{-9}$, which ensures both that the bound is respected and that numerical errors do not occur. For distributions with shift parameters, these parameters were also restricted to less than the sample minimum for CML fits and to less than the minimum quantile for QMP fits.

\section{Results}

Estimation failures. Only 50 fits out of the 1.35 million performed failed to produce usable parameter estimates, indicating that the starting point heuristics are robust. As is shown in Table 2, estimation of parameter standard errors and correlations failed at a greater (but still low) rate, because the Hessian was not invertible (i.e., not positive-definite). For brevity, Table 2 averages over parameter sets and sample sizes and omits results for the Gumbel, which never failed. Generally, better performance was obtained with larger samples and for more skewed distributions. QMP4 estimation for the Lognormal stands out as producing many more failures than the other cases, indicating that if parameter standard error and correlation estimates are required for the Lognormal, higher levels of grouping should be avoided.

Bias and efficiency. Bias was estimated as the difference between the mean of the Monte Carlo parameter estimates and the true value, with positive values indicating overestimation and negative values indicating underestimation. Efficiency was estimated by the $S D$ of the parameter estimates. Estimates are described as consistent if the magnitude of bias decreases, and efficiency increases as sample size increases. Tables $3-5$ contain the bias and efficiency estimates for the parameters of the shift distributions.

Table 2

Percentages of Fits With Invertible Hessian Estimates

\begin{tabular}{ccccc}
\hline Estimation Method & Ex-Gaussian & Lognormal & Wald & Weibull \\
\hline CML & 98.4 & 100.0 & 93.4 & 98.7 \\
QMP1 & 98.8 & 95.2 & 98.9 & 96.2 \\
QMP4 & 96.0 & 59.0 & 99.7 & 94.8 \\
\hline
\end{tabular}


To compare estimation performance across distributions, it is useful to recognize that the shift parameters $(\theta)$ and the Weibull scale parameter $(\tau)$ have the same units as the data. For these parameters, relative estimation performance can be judged on the same scale. Estimation performance can also be judged for all parameters as a proportion of their true values, which are given in Table 1.

The results for the ex-Gaussian distribution replicated Heathcote et al. (2002) with only a slightly different methodology (i.e., rounded samples from a different random number generator) and are omitted for the sake of brevity, as is a detailed discussion of the results for the Gumbel distribution, which were uniformly good in all cases and for all estimation methods. ${ }^{6}$ In contrast to the Gumbel and ex-Gaussian estimates, estimates for the shift distributions were very poorly behaved in some cases. That is, estimates were very biased and not always consistent (i.e., bias could increase and efficiency could decrease with sample size), and the parameter estimate distributions were not even approximately normal.

The Lognormal estimates were best behaved among the shift distributions in terms of consistency and distributions. Parameter estimate distributions were mainly unimodal, with the exception of CML estimates for the least skewed distribution at the smallest sample size, which had small second modes overestimating $\theta$ and underestimating $\mu$. For all estimation methods, the parameter estimate distributions were slightly skewed, particularly for small sample sizes, to the left for $\theta$ and to the right for $\mu$ and $\sigma$.

As is shown in Table 3, bias was generally upward for $\theta$ and $\sigma$ and downward for $\mu$. Bias in all parameters was substantial for the least skewed distribution, even for the largest sample, and small for the other two distributions at all sample sizes. The least skewed distribution has a small shift parameter (475) and a long left tail. As is evident from Figure 1, sampled values below 750 are rare, resulting in a strong upward bias in shift estimates even for larger sample sizes. Estimation efficiency and bias were consistent in all cases, except for a few cases occurring when bias was negligible, likely due to Monte Carlo error. CML was generally the least biased and QMP1, the most efficient.

For the Wald distribution, parameter estimate distributions were generally unimodal but could also be heavy tailed. All parameter estimate distributions contained extreme underestimates for shift and overestimates for $\mu$ and $a$, but the main body of the distribution tended to be skewed in the opposite direction, particularly for smaller samples and for CML. As is shown by the bias values in Table 4, the shift parameter of the least skewed Wald distribution was generally overestimated, whereas for the most skewed distribution it was generally underestimated. For the other parameters, the opposite pattern generally applied: underestimation for the least skewed distribution and overestimation for the most skewed distribution. Bias was particularly pronounced in CML estimates for the least skewed distribution. This bias was not due to extreme outliers; the same pattern occurred when the central tendency of the parameter estimate distribution was estimated from its median. Bias was consistent for all but CML estimates for the most skewed distribution and QMP4 estimates for the least skewed distribution, but the inconsistency was relatively small. Efficiency estimates were consistent, and CML estimates were clearly more efficient than QMP estimates. However, CML estimates were more biased than QMP1 estimates, particularly for the least skewed distribution. Hence, CML parameter estimate distributions are less variable but tend to be centered on a biased estimate of the true parameter value.

Table 3

Bias (Monte Carlo Mean - True Value) and Efficiency (SD) for Lognormal Parameter Estimates

\begin{tabular}{|c|c|c|c|c|c|c|c|c|c|c|c|}
\hline \multirow[b]{2}{*}{ Measure } & \multirow[b]{2}{*}{ Parameter } & \multirow{2}{*}{$\begin{array}{c}\text { Estimation } \\
\text { Method }\end{array}$} & \multicolumn{3}{|c|}{ Distribution 1} & \multicolumn{3}{|c|}{ Distribution 2} & \multicolumn{3}{|c|}{ Distribution 3} \\
\hline & & & 40 & 80 & 160 & 40 & 80 & 160 & 40 & 80 & 160 \\
\hline \multirow[t]{9}{*}{ Bias } & $\theta$ & CML & 131.1 & 128.8 & 103.1 & -2.4 & -1.8 & -3.4 & -5.1 & -1.3 & -1.5 \\
\hline & & QMP1 & 140.4 & 124.2 & 117.3 & -4.4 & -2.3 & -1.4 & -9.0 & -5.6 & -5.0 \\
\hline & & QMP4 & 171.3 & 142.4 & 118.8 & -3.5 & -3.5 & -2.3 & -16.9 & -8.7 & -5.1 \\
\hline & $\sigma$ & CML & 0.082 & 0.071 & 0.051 & 0.028 & 0.016 & 0.005 & 0.020 & 0.013 & 0.002 \\
\hline & & QMP1 & 0.091 & 0.072 & 0.063 & 0.029 & 0.015 & 0.007 & 0.015 & 0.004 & -0.004 \\
\hline & & QMP4 & 0.126 & 0.087 & 0.066 & 0.054 & 0.020 & 0.008 & 0.029 & 0.006 & -0.002 \\
\hline & $\mu$ & CML & -0.355 & -0.320 & -0.242 & -0.053 & -0.027 & -0.006 & -0.028 & -0.020 & -0.003 \\
\hline & & QMP1 & -0.373 & -0.310 & -0.283 & -0.037 & -0.019 & -0.008 & 0.003 & 0.011 & 0.018 \\
\hline & & QMP4 & -0.479 & -0.366 & -0.292 & -0.075 & -0.025 & -0.009 & 0.003 & 0.015 & 0.015 \\
\hline \multirow[t]{9}{*}{$S D$} & $\theta$ & CML & 120.8 & 84.8 & 70.4 & 75.7 & 57.6 & 45.0 & 54.9 & 36.4 & 22.4 \\
\hline & & QMP1 & 108.9 & 91.3 & 78.6 & 73.8 & 52.7 & 35.9 & 52.4 & 34.7 & 23.9 \\
\hline & & QMP4 & 116.5 & 99.3 & 87.5 & 91.0 & 62.2 & 41.6 & 75.5 & 45.1 & 27.5 \\
\hline & $\sigma$ & CML & 0.091 & 0.058 & 0.038 & 0.135 & 0.096 & 0.069 & 0.156 & 0.107 & 0.072 \\
\hline & & QMP1 & 0.088 & 0.061 & 0.045 & 0.128 & 0.089 & 0.061 & 0.150 & 0.104 & 0.074 \\
\hline & & QMP4 & 0.128 & 0.073 & 0.054 & 0.182 & 0.108 & 0.069 & 0.212 & 0.128 & 0.082 \\
\hline & $\mu$ & CML & 0.333 & 0.228 & 0.168 & 0.346 & 0.254 & 0.191 & 0.322 & 0.221 & 0.146 \\
\hline & & QMP1 & 0.313 & 0.239 & 0.193 & 0.328 & 0.234 & 0.162 & 0.309 & 0.213 & 0.152 \\
\hline & & QMP4 & 0.381 & 0.272 & 0.223 & 0.424 & 0.277 & 0.186 & 0.426 & 0.267 & 0.171 \\
\hline
\end{tabular}


Table 4

Bias (Monte Carlo Mean - True Value) and Efficiency (SD) for Wald Parameter Estimates

\begin{tabular}{|c|c|c|c|c|c|c|c|c|c|c|c|}
\hline \multirow[b]{2}{*}{ Measure } & \multirow[b]{2}{*}{ Parameter } & \multirow{2}{*}{$\begin{array}{l}\text { Estimation } \\
\text { Method }\end{array}$} & \multicolumn{3}{|c|}{ Distribution 1} & \multicolumn{3}{|c|}{ Distribution 2} & \multicolumn{3}{|c|}{ Distribution 3} \\
\hline & & & 40 & 80 & 160 & 40 & 80 & 160 & 40 & 80 & 160 \\
\hline \multirow[t]{9}{*}{ Bias } & $\theta$ & CML & 107.2 & 95.4 & 87.6 & 28.6 & 21.4 & 16.2 & -19.9 & -24.7 & -29.3 \\
\hline & & QMP1 & 15.0 & 7.3 & 3.4 & -15.3 & -10.9 & -6.7 & -21.8 & -18.4 & -17.3 \\
\hline & & QMP4 & -8.8 & 11.0 & 11.0 & -42.8 & -14.1 & -6.9 & -40.8 & -21.3 & -15.5 \\
\hline & $\mu$ & CML & -0.039 & -0.026 & -0.034 & -0.009 & -0.008 & -0.006 & 0.017 & 0.017 & 0.018 \\
\hline & & QMP1 & -0.013 & -0.009 & -0.004 & 0.003 & 0.003 & 0.003 & 0.012 & 0.010 & 0.009 \\
\hline & & QMP4 & -0.006 & -0.009 & -0.007 & 0.010 & 0.003 & 0.002 & 0.018 & 0.011 & 0.009 \\
\hline & $a$ & CML & -30.64 & -28.17 & -26.18 & -6.93 & -5.46 & -4.17 & 6.53 & 7.26 & 8.21 \\
\hline & & QMP1 & -5.45 & -2.96 & -1.14 & 5.28 & 3.80 & 2.62 & 6.85 & 5.27 & 4.67 \\
\hline & & QMP4 & 6.08 & -2.84 & -3.19 & 15.62 & 4.81 & 2.58 & 13.04 & 6.23 & 4.37 \\
\hline \multirow[t]{9}{*}{$S D$} & $\theta$ & CML & 31.9 & 26.2 & 21.7 & 27.8 & 20.8 & 15.0 & 19.4 & 13.1 & 9.9 \\
\hline & & QMP1 & 104.8 & 86.4 & 65.7 & 83.1 & 59.0 & 42.9 & 51.6 & 29.8 & 21.1 \\
\hline & & QMP4 & 167.0 & 101.7 & 75.6 & 144.3 & 74.0 & 48.5 & 94.6 & 42.6 & 24.9 \\
\hline & $\mu$ & CML & 0.018 & 0.012 & 0.009 & 0.020 & 0.013 & 0.010 & 0.022 & 0.015 & 0.011 \\
\hline & & QMP1 & 0.030 & 0.024 & 0.020 & 0.033 & 0.025 & 0.020 & 0.033 & 0.022 & 0.016 \\
\hline & & QMP4 & 0.048 & 0.031 & 0.022 & 0.048 & 0.029 & 0.021 & 0.044 & 0.026 & 0.017 \\
\hline & $a$ & CML & 7.16 & 5.18 & 4.24 & 7.29 & 5.00 & 3.78 & 5.86 & 3.86 & 2.90 \\
\hline & & QMP1 & 28.93 & 25.00 & 19.12 & 23.96 & 17.12 & 12.83 & 15.39 & 8.85 & 6.05 \\
\hline & & QMP4 & 53.45 & 30.81 & 22.46 & 46.85 & 21.92 & 14.15 & 31.21 & 12.92 & 6.95 \\
\hline
\end{tabular}

Bias was substantially smaller for the more skewed Weibull distributions. For these cases, QMP estimates, particularly QMP1 estimates, were less biased than CML estimates. For the least skewed distribution, CML estimates were substantially less biased than QMP estimates. However, even for CML, bias was substantial for the smallest sample size. For all distributions, the CML estimates were the most efficient, although the advantage over QMP1 was relatively small for the more skewed distributions, particularly at larger sample sizes. QMP4 was clearly the least efficient method, particularly for smaller sample sizes.

Weibull parameter estimate distributions were almost always bimodal to some degree for the least skewed case, although the second mode tended to disappear as sample size increased. The second mode always underestimated shift $(\theta)$ and overestimated the scale $(\tau)$ and shape $(c)$ parameters. Estimates fell in the second mode mainly for samples with negative Fisher skew. The Weibull distribution can have negative Fisher skew, which slowly approaches a bound of approximately -1.14 for large values of $c$. For example, Fisher skew values are -0.08 , $-0.6,-1,-1.1$, and -1.13 for $c=4,10,100,1,000$, and 10,000 , respectively. These results indicate that caution should be exercised when Weibull is fitted to samples with negative Fisher skew.

Estimates in the deviant mode usually had noninvertible Hessians, indicating that the neighborhood of the

Table 5

Bias (Monte Carlo Mean - True Value) and Efficiency (SD) for Weibull Parameter Estimates

\begin{tabular}{|c|c|c|c|c|c|c|c|c|c|c|c|}
\hline \multirow[b]{2}{*}{ Measure } & \multirow[b]{2}{*}{ Parameter } & \multirow{2}{*}{$\begin{array}{c}\text { Estimation } \\
\text { Method }\end{array}$} & \multicolumn{3}{|c|}{ Distribution 1} & \multicolumn{3}{|c|}{ Distribution 2} & \multicolumn{3}{|c|}{ Distribution 3} \\
\hline & & & 40 & 80 & 160 & 40 & 80 & 160 & 40 & 80 & 160 \\
\hline \multirow[t]{9}{*}{ Bias } & $\theta$ & CML & -25.8 & -9.2 & -3.1 & 4.7 & 5.2 & 4.0 & 6.7 & 4.4 & 2.7 \\
\hline & & QMP1 & -90.6 & -46.6 & -17.5 & -3.6 & 0.2 & 1.2 & 2.2 & 1.8 & 1.0 \\
\hline & & QMP4 & -95.9 & -95.0 & -44.5 & -28.1 & -3.8 & 1.9 & -5.0 & 1.8 & 1.6 \\
\hline & $\tau$ & CML & 24.4 & 8.3 & 2.6 & -6.9 & -6.8 & -5.0 & -9.6 & -6.6 & -3.9 \\
\hline & & QMP1 & 91.2 & 47.3 & 17.9 & 0.4 & -2.0 & -2.9 & -7.1 & -6.2 & -5.0 \\
\hline & & QMP4 & 94.9 & 96.5 & 45.3 & 25.5 & 2.0 & -3.5 & 1.8 & -4.6 & -4.1 \\
\hline & $c$ & CML & 0.47 & 0.17 & 0.06 & 0.01 & -0.03 & -0.04 & -0.06 & -0.05 & -0.03 \\
\hline & & QMP1 & 1.15 & 0.58 & 0.21 & 0.07 & 0.01 & -0.02 & -0.02 & -0.03 & -0.02 \\
\hline & & QMP4 & 1.25 & 1.14 & 0.52 & 0.40 & 0.06 & -0.02 & 0.10 & -0.02 & -0.02 \\
\hline \multirow[t]{9}{*}{$S D$} & $\theta$ & CML & 160.6 & 93.9 & 47.0 & 44.9 & 20.5 & 11.5 & 14.4 & 7.1 & 4.1 \\
\hline & & QMP1 & 251.7 & 168.7 & 78.0 & 68.5 & 22.7 & 13.1 & 22.2 & 8.0 & 4.6 \\
\hline & & QMP4 & 299.8 & 258.7 & 171.6 & 166.8 & 63.4 & 20.8 & 73.3 & 13.7 & 6.7 \\
\hline & $\tau$ & CML & 167.0 & 98.8 & 50.7 & 53.9 & 27.7 & 16.6 & 26.4 & 16.3 & 10.9 \\
\hline & & QMP1 & 262.1 & 175.7 & 81.6 & 76.7 & 30.5 & 19.3 & 32.1 & 17.4 & 11.7 \\
\hline & & QMP4 & 308.9 & 268.1 & 177.9 & 173.8 & 68.8 & 26.0 & 78.8 & 21.1 & 12.4 \\
\hline & $c$ & CML & 2.24 & 1.25 & 0.62 & 0.70 & 0.34 & 0.20 & 0.33 & 0.18 & 0.11 \\
\hline & & QMP1 & 3.24 & 2.11 & 0.97 & 0.97 & 0.36 & 0.22 & 0.39 & 0.18 & 0.12 \\
\hline & & QMP4 & 3.86 & 3.14 & 2.02 & 2.22 & 0.84 & 0.30 & 1.10 & 0.25 & 0.14 \\
\hline
\end{tabular}



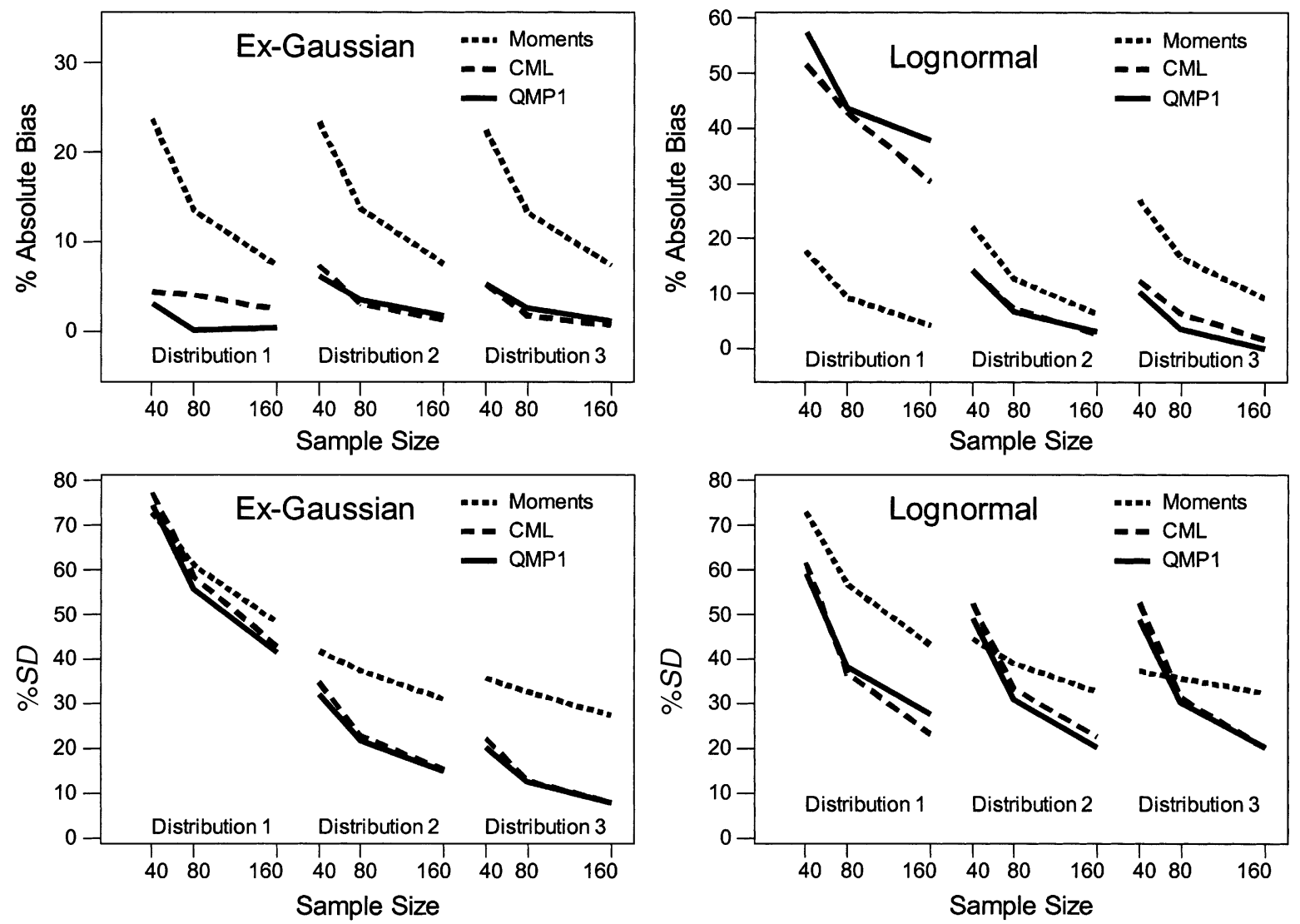

Figure 2. Mean absolute bias and efficiency $(S D)$ for Fisher skew estimates, as a percentage of the true value, estimated using the method of moments, CML, and QMP1.

solution is not locally quadratic. When estimates with nonpositive-definite Hessians were censored, bias was reduced although not eliminated. Hence, it appears that censoring estimates with ill-conditioned Hessians can improve overall estimation performance for the Weibull. An alternative approach is to bound the estimate of $c$ during estimation, a strategy that can be easily implemented by modifying and recompiling the QMPE opensource code. We obtained improved bias and efficiency using an upper bound of 10 , but this resulted in even more clearly bimodal parameter estimate distributions with estimates "piling up" against the bound.

Estimating Fisher skew. Ratcliff and Murdock (1976) used CML to fit the ex-Gaussian distribution in order to estimate RT distribution skew. Ratcliff (1978) pointed out that estimates of skew based on the method of moments are both inefficient and nonrobust. Hence, unrealistically large sample sizes are required for precise estimates, and estimates can be greatly distorted by even small levels of outlier contamination. In this section, we compare estimates obtained by the indirect method of calculating Fisher skew from CML and QMP1 parameter estimates with those obtained directly from the method of moments. Skew estimates for all three-parameter distributions (i.e., those with variable skew) are shown in Figure 2. Results are ex- pressed as a percentage of the true Fisher skew value, and absolute values of bias are given in order to make comparison easier.

For the ex-Gaussian distribution, both CML and QMP1 estimates were less biased and more efficient than method of moments estimates, with QMP1 estimates having clearly less bias than CML estimates for the least skewed distribution. Efficiency was comparable for CML and QMP1 and clearly better than for method of moments except for the least skewed distribution and smallest sample size. For the Lognormal, the method of moments estimates were much less biased than the CML and QMP1 estimates for the least skewed distribution, but more biased for the more skewed distributions. Generally, the method of moments estimates are less efficient than the CML and QMP1 estimates, except for the smallest sample size and more skewed distributions.

For the Wald distribution, the method of moments estimates were least efficient and the CML estimates most efficient. However, CML estimates were the most biased, particularly for the least skewed distribution, and QMP1 estimates were the least biased overall. For the least skewed Weibull distribution, the method of moments estimates were the least biased and most efficient, although all estimation methods displayed poor efficiency due to 

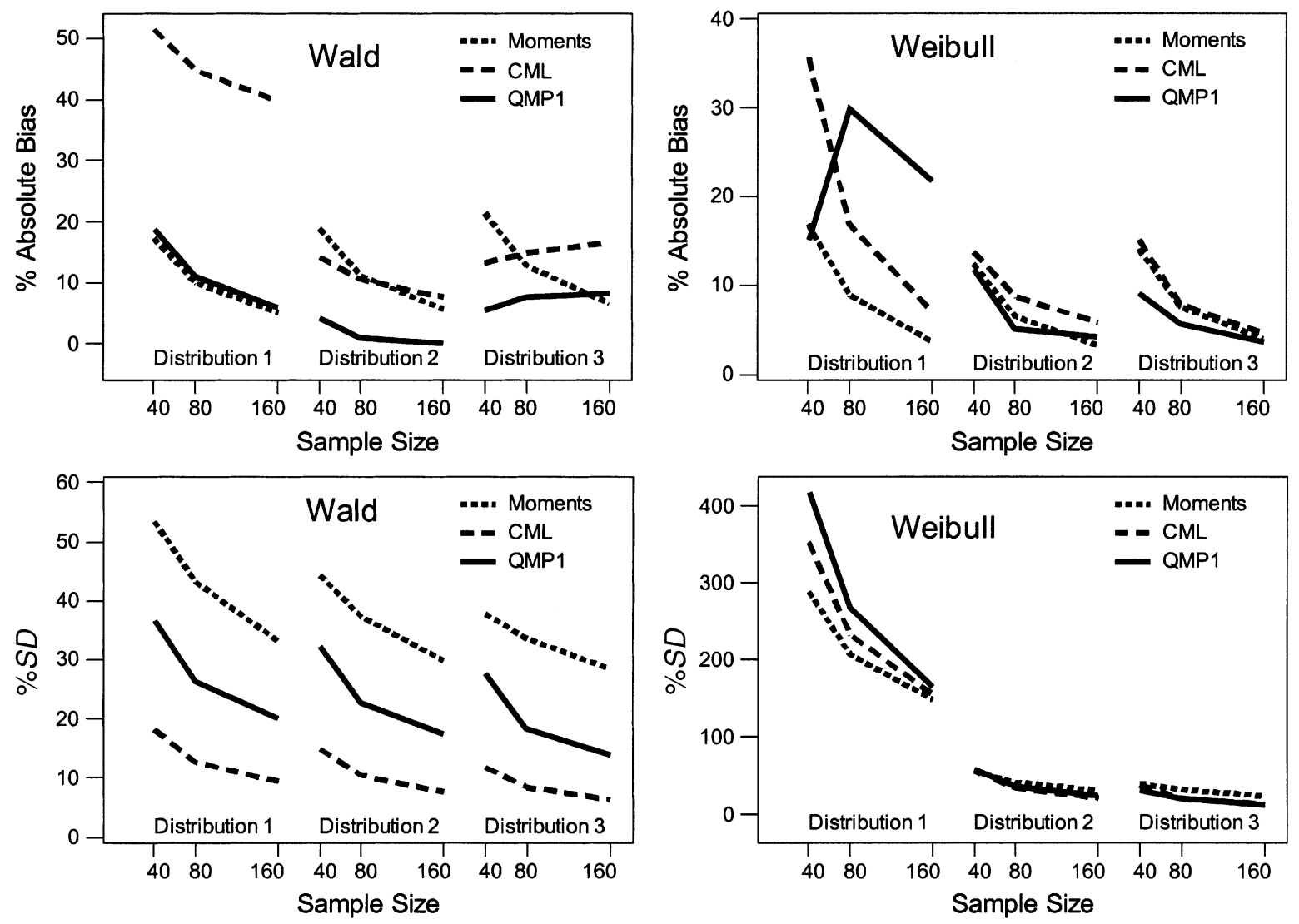

Figure 2 (Continued).

bimodal parameter estimate distributions. Efficiency problems may have been exaggerated by the use of a percentage measure, since Fisher skew was smaller for the Weibull than for other distributions in the least skewed case. Estimation performance was much better for the more skewed distributions, with QMP1 the best overall.

The results presented in Figure 2 provide a basis for comparing estimation performance among distributions with variable skew. Clearly, CML and QMP1 fits of the ex-Gaussian distribution provide better skew estimates than the method of moments at all levels of skew. QMP1 and CML are also generally superior for the two more skewed cases of the other distributions. For the least skewed case, however, the method of moments generally outperforms QML1 and CML, reflecting the estimation difficulties for this case noted in the last section.

\section{Discussion}

The results of the Monte Carlo study confirmed that QMP is generally superior to CML for the two distributions with an unbounded range: the ex-Gaussian and the Gumbel. CML and QMP are on a more equal footing for the shift distributions when Fisher skew is greater than one, although it should be remembered that for more extreme skew CML can fail entirely for the Lognormal and
Weibull distributions, due to the unbounded likelihood problem.

No method worked very well for the least skewed Lognormal, Wald, and Weibull distributions, particularly for smaller sample sizes. Overestimation of shift for the Lognormal and Wald distributions results from their long, thin left tails in the least skewed cases. Overestimation in these cases may be difficult to avoid, because information about the shift value is quite variable. Hence, QMPE parameter estimates for these distributions should be interpreted with caution when Fisher skew is less than one.

Underestimation of shift for the Weibull appears to be related to a nonquadratic maximum for both CML and QMP, as is indicated by ill-conditioned Hessian estimates. Removal of cases with ill-conditioned Hessians improved performance. Heathcote (in press) noted similar behavior for CML estimates of Wald distributions in small samples $(n=40)$ and suggested that estimates with ill-conditioned Hessians should be censored when his software is used. However, censoring QMPE Wald estimates with ill-conditioned Hessians did not reduce bias appreciably. In contrast, when Heathcote's (in press) optimization methods (the S-plus nlminb algorithm using analytic first and second derivatives) were applied to the Wald data from the present Monte Carlo study, bias was 
almost eliminated by censoring. Unfortunately, reduced bias was bought at the cost of reduced efficiency relative to the QMPE estimates.

Cheng and Iles (1990) provide an explanation for these difficulties, called the embedded models problem. They showed that each of the shift distributions fit by QMPE has a special case as shift approaches $-\infty$, which they call an embedded distribution. The embedded distributions (normal for all but the Weibull, which has an embedded left skewed Gumbel distribution) have only two parameters: a scale parameter and a location parameter. When the embedded model fits as well as the shift model, this indicates that the sample does not contain sufficient information to estimate shift or shape; instead, only location and scale can be reliably estimated. Because the embedded model occurs at an infinite parameter value, iterative estimation of the shift model is difficult, and estimates for all of its parameters become unreliable.

The bimodality and underestimation of shift seen in the QMPE Weibull parameter estimates appears to be due to the embedded model problem. Similar problems found here for some QMP4 estimates produced by QMPE and by Heathcote (in press) for CML estimates of the shifted Wald appear to be examples of the embedded model problem. Heathcote's (in press) Wald estimates were probably more prone to this problem than QMPE Wald estimates because his fitting routine used analytic Hessians, and so was more sensitive to the nonquadratic maximum produced by the embedded model problem.

\section{GENERAL DISCUSSION}

In this article, we have described and tested QMPE, an open-source ANSI standard Fortran 90 program, which can estimate the parameters of five continuous density functions commonly used to model RT. QMPE can fit these distributions using continuous maximum likelihood (CML), perhaps the most widely used and recommended method of estimating RT distribution (Heathcote, 1996; Van Zandt, 2000). It can also fit these distributions using Heathcote et al.'s (2002) QMP method.

A Monte Carlo study replicated Heathcote et al.'s (2002) finding that QMP produces less biased and more efficient parameter estimates than CML does for the ex-Gaussian distribution. QMP was also found to produce less biased, but also slightly less efficient, parameter estimates than CML does for the Gumbel distribution. Overall estimation performance was excellent for both distributions, as might be expected in the idealized situation represented by the Monte Carlo study, fitting the true data-generating model to uncontaminated data. Of course, real RT data do not conform to this ideal, but at least the excellent performance under ideal conditions is reassuring for the practical application of QMPE.

The ex-Gaussian and Gumbel distributions have an unbounded range. This might be seen as a disadvantage when they are used to model RT data, because RT data must be bounded below by a positive value. QMPE also fits three shift distributions, which have a positive, parameterdependent lower bound, and so seem more promising as models of RT. Unfortunately, estimation performance for the shift distributions was much worse than for the exGaussian or the Gumbel. One reason for fitting parametric distributions to RT data is to obtain a more reliable estimate of skew than is provided by the method of moments (Ratcliff, 1978). If QMPE is used for this purpose, we suggest that the ex-Gaussian be fit (the Gumbel has fixed skew and so is not useful for this purpose). At least when the ex-Gaussian is an accurate approximation to the data, this approach is more efficient and less biased than the method of moments. The shift distributions, in contrast, did not consistently outperform the method of moments, and for less skewed distributions and small sample sizes they could produce substantially worse skew estimates.

One reason for which the ex-Gaussian outperforms the shift distributions might be the parameterizations used by QMPE and most packages aimed at fitting RT distribution (see Cousineau, Brown, \& Heathcote, 2004). The ex-Gaussian has its least skewed (Gaussian) form when its exponential parameter approaches zero. The shift distributions have their least skewed form when the shift parameter approaches $-\infty$. In small samples, the least skewed case may often be most appropriate because the data contain mainly information about location and scale. Because this case occurs as the shift parameter diverges, parameter estimates for the shift distributions become unreliable, whereas this does not happen for the ex-Gaussian.

Cheng and Iles (1990) suggested solving this problem, which they dubbed the embedded model problem, by using a parameterization of the shift distributions in which the least skewed case occurs at zero rather than at an infinite value of one parameter (see their Table 1). We have implemented and tested Cheng and Iles's (1990) parameterization in a new version of the QMPE software. However, little improvement in estimation performance was obtained, even following censoring of cases with negative shift estimates or those in which the full model did not fit significantly better than the embedded model. We suggest that users of the existing version of QMPE and similar software exercise caution in interpreting parameter estimates from small samples, particularly those with negative skew or Hessians that are not invertible. We are currently investigating the use of hierarchical models to address these difficult cases. Hierarchical models implement "soft bounding" on parameter estimates by assuming that parameters are drawn from a population distribution with an assumed form (see Rouder, Lu, Speckman, Sun, \& Jiang, in press, for a Bayesian approach to hierarchical Weibull estimation).

The embedded model problem occurs for data with low skew. Highly skewed data can also cause a problem, called the unbounded likelihood problem, for CML estimation of the shifted Weibull and Lognormal distributions. The problem arises because the likelihood maximum occurs when the shift parameter equals the minimum 
observed value. Although this might seem like a plausible estimate of shift, estimates of the remaining parameters are inconsistent. QMP does not suffer from the unbounded likelihood problem and so remains useful for highly skewed data. Hence, when skew is high, we recommend QMP fitting over CML fitting. QMP might also be useful in other contexts, such as fitting mixtures (see, e.g., Dolan, van der Maas, \& Molenaar, 2002), since they can also be subject to the unbounded likelihood problem (Cheng \& Traylor, 1995).

In the course of this investigation, we discovered that QMP has a special case called the maximum of product spacings (MPS), which was advocated by Cheng and Amin (1983) and by Ranneby (1984) as a means of overcoming the unbounded likelihood problem. Their work proved that MPS has all of the desirable asymptotic properties of CML when CML estimates exist and continues to work well when CML fails. Further consideration of the MPS and its generalizations (see, e.g., Ekström, 2001) is beyond the scope of the present work. However, this literature places QMP on a firm theoretical footing, not just as an approximation to likelihood but also as a goodness-offit measure that can be derived from information theory (see also Heathcote \& Brown, in press; Speckman \& Rouder, in press).

\section{REFERENCES}

Brown, S., \& Heathcote, A. (2003). QMLE: Fast, robust, and efficient estimation of distribution functions based on quantiles. Behavior Research Methods, Instruments, \& Computers, 35, 485-492.

BReukelen, G. J. P. (1995). Parallel information processing models compatible with lognormally distributed response times. Journal of Mathematical Psychology, 39, 396-399.

Cheng, R. C. H., \& Amin, N. A. K. (1981). Maximum likelihood estimation of parameters in the inverse Gaussian distribution with unknown origin. Technometrics, 23, 257-263.

Cheng, R. C. H., \& Amin, N. A. K. (1983). Estimating parameters in continuous univariate distributions with a shifted origin. Journal of the Royal Statistical Society: Series B, 45, 394-403.

Cheng, R. C. H., \& Iles, T. C. (1987). Corrected maximum likelihood in nonregular problems. Journal of the Royal Statistical Society: Series $B, \mathbf{4 9}, 95-101$.

Cheng, R. C. H., \& ILes, T. C. (1990). Embedded models in threeparameter distributions and their estimation. Journal of the Royal Statistical Society: Series B, 52, 135-149.

Cheng, R. C. H., \& TraYlor, L. (1995). Non-regular maximum likelihood problems. Journal of the Royal Statistical Society: Series B, 57, 3-44.

Cousineau, D., Brown, S., \& Heathcote, A. (2004). Fitting distributions using maximum likelihood: Methods and packages. Manuscript submitted for publication.

Cousineau, D., Goodman, V., \& Shiffrin, R. M. (2002). Extending statistics of extremes to distributions varying on position and scale, and implication for race models. Journal of Mathematical Psychology, 46, 431-454.

Dolan, C. V., van der Maas, H. L. J., \& Molenaar, P. C. M. (2002). A framework for ML estimation of parameters (mixtures of) common reaction time distributions given optional truncation or censoring. Behavior Research Methods, Instruments, \& Computers, 34, 304-323.

ЕкSTRÖм, M. (2001). Consistency of generalized maximum spacing estimates. Scandinavian Journal of Statistics, 28, 343-354.

Giesbrecht, F., \& Kempthorne, O. (1976). Maximum likelihood estimation in the three-parameter lognormal distribution. Journal of the Royal Statistical Society: Series B, 38, 257-264.
Gumbel, E. J. (1958). The statistics of extremes. New York: Columbia University Press.

HEATHCOTE, A. (1996). RTSYS: A DOS application for the analysis of reaction time data. Behavior Research Methods, Instruments, \& Computers, 28, 427-445.

HeathCote, A. (in press). Fitting the Wald and ex-Wald distributions to response time data. Behavior Research Methods, Instruments, \& Computers.

HeAthCote, A., \& Brown, S. (in press). Reply to Speckman and Rouder: A theoretical basis for QML. Psychonomic Bulletin \& Review.

Heathcote, A., Brown, S., \& Mewhort, D. J. K. (2002). Quantile maximum likelihood estimation of response time distributions. Psychonomic Bulletin \& Review, 9, 394-401.

Hyndman, R. J., \& FAN, Y. (1996). Sample quantiles in statistical packages. American Statistician, 50, 361-365.

McClelland, J. L. (1979). On the time relations of mental processes: An examination of systems of processes in cascade. Psychological Review, 86, 287-330.

McGiLL, W. J. (1963). Stochastic latency mechanisms. In R. D. Luce, R. R. Bush, \& E. Galanter (Eds.), Handbook of mathematical psychology (pp. 193-199). Oxford: Wiley.

RANNEBY, B. (1984). The maximum spacing method: An estimation method related to the maximum likelihood method. Scandinavian Journal of Statistics, 11, 93-112.

RATCLIFF, R. (1978). A theory of memory retrieval. Psychological Review, 85, 59-108.

RATCLIFF, R., \& MuRdock, B. B. (1976). Retrieval processes in recognition memory. Psychological Review, 83, 190-214.

Rouder, J. N., Lu, J., SPeckman, P., Sun, D., \& Jiang, Y. (in press). A hierarchical model for estimating response time distributions. Psychonomic Bulletin \& Review.

SPeCKMan, P. L., \& Rouder, J. N. (in press). A comment on Heathcote, Brown, and Mewhort's QMLE estimation method for response time distributions. Psychonomic Bulletin \& Review.

Titterington, D. M. (1985). Comment on "Estimating parameters in continuous univariate distributions." Journal of the Royal Statistical Society: Series B, 47, 115-116

UlRich, R., \& MilLER, J. (1994). Effects of outlier exclusion on reaction time analysis. Journal of Experimental Psychology: General, 123, 34-80.

WALD, A. (1947). Sequential analysis. New York: Wiley.

WeIBULL, W. (1951). A statistical distribution function of wide applicability. Journal of Applied Mechanics, 18, 292-297.

West, B. J., \& Shlesinger, M. (1990). The noise in natural phenomena. American Scientist, 78, 40-45.

WoodWorth, R. S., \& SCHLOSBERG, H. (1954). Experimental psychology. New York: Holt.

VAN ZANDT, T. (2000). How to fit a response time distribution. Psychonomic Bulletin \& Review, 7, 424-465.

\section{NOTES}

1. Source code, Linux and Windows binaries, a manual, and sample instruction and data files can be obtained from either of the second and first authors' Web sites (http://oz.ss.uci.edu/ and http://www.newcastle.edu.au/ school/behav-sci/ncl/). These supplementary materials have also been accepted for the Psychonomic Society Norms, Stimuli, and Data Archive, available after August 1, 2004 at http://www.psychonomic.org.

2. Heathcote et al. (2002) described their method as quantile maximum likelihood (QML). Speckman and Rouder (in press) pointed out that QML is not maximum likelihood, so Heathcote and Brown (in press) renamed the method quantile maximum probability (QMP).

3. This parameterization is convenient when shift does not have to be estimated, since analytic maximum likelihood estimates are available for both the arithmetic mean and

$$
\frac{1}{\hat{\lambda}}=\frac{\sum_{i=1}^{n}\left(x_{i}^{-1}-\hat{\kappa}_{1}^{-1}\right)}{n} .
$$

When shift is estimated, iterative methods are required, but computational cost can be reduced using profile likelihood (a line search on the shift parameter, with other parameters estimated analytically). 
4. We reserve the term $Q M P$ for the general procedure that maximizes Equation (1) on the basis of a set of quantile estimates obtained in an unspecified manner. Many different quantile estimators are available (see Hyndman \& Fan, 1996, for a review). Although these estimators are asymptotically equivalent, they differ in finite samples and so can result in differing parameter estimates in practice. In the QMPE software, we implemented the quantile estimator specified in Heathcote et al. (2002), which produces QMP1 and QMP4 estimates as special cases and corresponds in general to Hyndman and Fan's Definition 5. Although we have found that this estimator works well, QMPE can be used with other quantile estimators if the estimated quantile values, rather than raw data, are provided as input to the program.

5. By default, S-plus has functions to generate samples from all distributions fit by QMPE except the Wald. Wald random deviates were obtained using an S-plus function that uses the inverse Gaussian param- eterization (available at http://www.statsci.org/s/invgauss.s). Heathcote (in press) provides an S-plus Wald random-number generator using the diffusion parameterization.

6. All estimation methods were consistent for the Gumbel, with negligible bias and good efficiency $(S D<15)$ for all sample sizes, estimation methods, and parameters, with a slight improvement for the less variable distributions. QMP was less biased than CML, but in contrast to findings with the ex-Gaussian distribution, QMP4 was less biased than QMP1. CML and QMP1 were almost equal in efficiency, with QMP4 being slightly less efficient in some cases. The results indicate that all three methods should be useful in practice with samples as small as 40 observations and perhaps fewer.

(Manuscript received May 6, 2003 ;

revision accepted for publication April 22, 2004.) 